\title{
Chronique bibliographique. Une police pure : la lutte contre la criminalité organisée vue par Norbert Pütter
}

\section{(2) OpenEdition \\ Journals}

\section{Édition électronique}

URL : http://journals.openedition.org/conflits/286

DOI : $10.4000 /$ conflits.286

ISSN : $1777-5345$

Éditeur :

CCLS - Centre d'études sur les conflits lilberté et sécurité, L'Harmattan

Édition imprimée

Date de publication : 1 juin 2000

ISBN : 2-7384-9946-5

ISSN : 1157-996X

Référence électronique

"Chronique bibliographique. Une police pure : la lutte contre la criminalité organisée vue par Norbert Pütter », Cultures \& Conflits [En ligne], 38-39 | été-automne 2000, mis en ligne le 20 mars 2006,

consulté le 30 mars 2021. URL : http://journals.openedition.org/conflits/286 ; DOI : https://doi.org/ $10.4000 /$ conflits.286

Ce document a été généré automatiquement le 30 mars 2021.

Creative Commons License 


\section{Chronique bibliographique. Une police pure : la lutte contre la criminalité organisée vue par Norbert Pütter}

1 Am Anfang war kein Unrecht Niklas Luhmann

2 Par sa nature même, la police s'oppose, on le sait, au "projet de connaître ${ }^{1}$. Ce constat, de ceux les mieux partagés par les sociologues de la police, a toutefois le tort de mettre sous silence l'inégale intensité de l'activité savante sur la police. Car le contrôle exercé par l'institution sur la visibilité des données, s'il n'empêche ou ne censure la production savante ${ }^{2}$, en dessine parfois les contours. Un indicateur fiable de cette logique spécifique de la production sociologique sur la police est l'opacité croissante de la connaissance à mesure que le sociologue s'éloigne de l'activité de sécurité publique pour aborder l'activité de police judiciaire. L'un des objets de la sécurité publique consiste à assurer la plus grande présence effective de la police auprès des citoyens afin d'établir, dans la durée, la manifestation d'une relation de «service public» à l'égard des « usagers ». La visibilité de ces programmes est leur ambition même et offre ainsi matière abondante au sociologue ${ }^{3}$. Et lorsque de surcroît la police estime disposer en telle ou telle partie de son magistère d'activités d'excellence, la sociologie se fait non seulement plus abondante, mais elle peu s'offrir le luxe de constructions causales fondées sur des comparaisons chiffrées, nourries d'observations, d'entretiens, de dépouillement d'archives, de documents administratifs internes, etc. Par ses manifestations, l'objet s'offre alors à une sociologie durkheimienne des causes et des correspondances. En revanche, là où le secret empreint le travail quotidien des agents, là où l'action se fait "sale boulot " 4 , là où les taux d'élucidation rongent la grandeur du travail policier, l'institution se fait plus réservée, et la recherche académique plus rare. Mais on observe également un déplacement des modèles explicatifs convoqués, ce qui est plus surprenant. L'institution policière indexe en effet trop volontiers la confidentialité des données qui permettent sa connaissance à la crainte de la défaveur qu'elles attireraient sur elle. Il 
n'est donc pas étonnant de constater que les missions de police "secrète » le restent d'autant mieux à l'égard des chercheurs que leur efficacité souffre d'une assise suffisante. Cependant, soumise à l'impératif de justification de son existence et de ses moyens, privant le public de matériaux visibles, la police doit abonder de discours assurant la légitimité des fins de son action. Par économie contrainte, ou faisant de nécessité vertu, le sociologue s'attache alors avec d'autant plus d'aisance à la sociologie des constructions de la réalité échafaudées par l'institution ${ }^{5}$. Mais alors, ne se donnant pas les moyens de tester l'hypothèse selon laquelle ces discours qui entourent, accompagnent et finalement recouvrent d'un voile opportun ces pratiques, et d'en épuiser la compréhension, il inverse le processus hypothetico-déductif de recherche et ne voit dans ces pratiques policières que les discours dont elles sont parées; alors même que fondées à poursuivre des réalités construites, ces pratiques constituent des réalités tangibles, et en effet souvent bien sales, pour ceux qui les mettent en œuvre et pour ceux qui en sont les cibles. Nouveaux domaines de l'action policière, les formes de " criminalité non apparente » réclament de l'activité de police judiciaire secret, silence, enquêtes sous couverture, technologies de pointe... qui rendent les pratiques plus opaques et redoublent la dépendance de l'observateur à l'égard de l'institution qui lui octroie ses tickets d'entrée. Travailler sur les activités spécialisées de police judiciaire se pose ainsi d'emblée comme une épreuve de résistance à l'inclination contrainte au constructivisme, épreuve qui enjoint de mobiliser une longue et durable connaissance des instruments juridiques, techniques et organisationnels de l'activité étudiée. Der OKKomplex, de Norbert Pütter, tire toute sa force du succès remporté dans cette épreuve. Contrairement à ce que le titre suggère ${ }^{6}$, l'objet du livre est la mutation accélérée des dispositifs policiers et pénaux sous l'effet de la lutte contre la criminalité organisée (ciaprès « LCO »). L'ouvrage de Pütter est à rebours de la littérature sociologique en vogue sur la criminalité dite « non apparente ». L'idée selon laquelle la criminalité organisée est une réalité construite n'est pas la finalité de l'ouvrage, mais son point de départ. La seule source de connaissance (savante et commune) sur la «CrimOrg » est la police (p. 10). Cette dernière ne s'en cache pas: elle avance sans crainte que la substance même de son travail en la matière se nourrit d'une seule hypothèse, l'invisibilité du phénomène: "La criminalité organisée, et ceci est admis avec d'autant plus de franchise que l'on est convaincu de son existence, n'est pas visible. Elle est cachée. La criminalité organisée s'offre seulement à la vue quand elle bat de l'ail'. Mais dans les cas ordinaires, elle n'existe et ne se développe que de manière invisible, sous la surface des activités sociales ordinaires et légales»(p. 9). Le crime n'est donc pas la manifestation du phénomène, mais de sa déliquescence, la manifestation, donc, de sa probable extinction (règlements de compte, assassinats de mauvais payeurs, entrée en scène de seconds couteaux, etc.). Travailler à démontrer le travail de légitimation par la police de l'objet est donc du temps perdu: que l'objet soit un construit constitue la seule connaissance accessible sur l'objet. Travailler à comprendre les instruments juridiques, techniques et organisationnels qui permettent l'institution d'une police de "lutte contre la criminalité organisée ", la codification juridique étroite d'un phénomène invisible et l'imbrication des champs policiers, judiciaires et politiques autour de cet invisible, tels sont les objets de ce livre. Le discours policier sur la criminalité organisée n'est qu'un élément anecdotique, souvent étonnant de franchise ; il n'est mobilisé dans la démonstration qu'à titre supplétif. Car l'ouvrage s'appuie sur des instruments d'enquête dont la diversité, la solidité et le mode d'exploitation tranchent au regard de la substance vaporeuse de l'objet, à laquelle il refuse de se 
soumettre : questionnaire ouvert ( $\mathrm{n}=25)$, disponible en annexe, adressé aux 75 policiers et procureurs de six Länder (de l'Ouest) ; 71 interviews menées avec les destinataires du questionnaire, d'une durée de $40 \mathrm{mn}$ à $21 / 2$ heures $^{8}$; questionnaire fermé, disponible en annexe, administré pour vérification des données d'entretien auprès de policiers relevant de divers services de police judiciaire (dont ceux de lutte contre la criminalité organisée) ; recherche de documents législatifs (donc publics) et administratifs (donc confidentiels) sur la LCO. Enfin, l'enquête et la rédaction, d'une durée de cinq ans, emportent si bien la démonstration que l'auteur se fait muet sur toutes les implications de sociologie théorique portées par son enquête : sa rigueur peut en effet s'autoriser le luxe de se passer du superflu. Il revient au lecteur, comme nous le ferons ici, de dégager les lignes théoriques fortes dessinées par l'ouvrage. Peu de discours, donc, mais des faits. Car si la police produit du discours en matière de LCO, elle n'est pas avare de pratiques. Décrites dans les moindres détails, comme c'est le cas dans l'ouvrage, elles manifestent la consécration, inaugurée bien sûr longtemps avant l'invention de la notion de CrimOrg 9 , de propriétés sectorielles fortes de la police judiciaire. Fortement encouragés par la LCO, ces propriétés sont à lire dans les modes d'enquête policière, dans les répercussions des revendications des agents des services de LCO sur la loi pénale et dans les mutations de la police de renseignement. En ce qui concerne les pratiques d'enquête, les traits structurels connus ${ }^{10}$ de l'autonomie de l'action de police judiciaire, se trouvent décuplés par la nature construite de la CrimOrg. L'enquête de police classique s'attache aux causes des phénomènes, par une double méthode descriptive (" constater les infractions ») et déductive ( rassembler les preuves », pour les organiser en vue de découvrir les "auteurs ") ${ }^{11}$. Cette démarche tranquillement inductive, qui remonte la chaîne causale depuis le fait commis jusqu'à son auteur, est balayée ici par la nature invisible de la CrimOrg, invisibilité qui touche tant l'essence du phénomène (l'organisation n'est jamais apparente) que les manifestations du phénomène (la criminalité est dite "sans victime apparente » - cf. infra). En ce sens, elle serait la forme la plus achevée de ces curieux délits sans autre victime que ses auteurs, selon une logique que la répression de l'usage de stupéfiants a déjà illustrée ${ }^{12}$. La Crimorg réclame donc une enquête policière d'une tout autre nature que celle qui s'attache à rechercher la cause d'actes donnés. Puisqu'on ne peut partir du délit commis pour en retrouver l'auteur, on infiltre tous les milieux où sont susceptibles de se terrer les auteurs possibles, pour tenter de déterminer si l'entrelacs de leurs activités (licites ou illicites) peut prendre la forme d'une organisation criminelle. Ainsi, la LCO exige une connaissance par infiltration, par immersion, de longue durée, centrée sur des groupes qui ont pour propriété de ne pas avoir commis de crime visible. Ensuite, puisque la structure criminelle peut simplement exister des semaines, voire des mois entiers, sans commettre aucun délit d'importance, l'enquête qui vise à en décrire le fonctionnement et les ramifications n'a pas, elle non plus, de fin. Autrement dit, la LCO rassemble tout un ensemble de propriétés étonnantes. Son déclenchement est indépendant des circonstances: la police décide du début de l'enquête (pp. 32-58). Puisqu'aucun délit n'est commis, puisque la Crimorg ne se découvre pas à partir de formes déterminées de manifestation, la police décide aussi des objets de l'enquête et des groupes à connaître (pp. 58-112). Puisque l'objet de l'enquête est la mise au jour de structures durables, et non d'événements ponctuels, l'enquête procède par immersion sur la durée (au moyen d'informateurs - "V-Männer " ${ }^{13}$, ou bien d'agents sous couverture - «verdeckte Ermittler »). Puisque l'objet de l'enquête est la mise au jour d'activités, et non pas des causes d'un acte déterminé, l'enquête n'est pas limitée dans 
sa matière (pp. 154-158) ${ }^{14}$. Autant le dire, ce type d'action policière se pose en contradiction à un principe fondamental de l'État de droit et à deux pierres angulaires de la procédure pénale : principe de légalité, opportunité des poursuites et domaine de l'enquête judiciaire. L'infraction au principe de légalité est évidente : s'il n'est point de crime, pourquoi une enquête ? C'est donc que l'enquête est préventive ${ }^{15}$. En ce cas, afin qu'elles échappent à l'arbitraire policier, (la police judiciaire est simple instrument, il faut le rappeler, de l'autorité judiciaire), ces enquêtes sont d'ordinaire étroitement contrôlées par le procureur. Mais voilà : la CrimOrg est une chose trop sérieuse, trop inquiétante et trop puissante pour être laissée à l'appréciation des procureurs. Ces derniers sont totalement dépourvus de moyens de contrôle, notamment dans les premières phases de l'enquête (pp. 227-261). S'ils manifestent une quelconque volonté de contrôle de l'activité policière, les policiers maintiennent leur dispositif de surveillance, et n'en informent plus personne, jusqu'au délit... s'il survient! (p. 209) Cette soudaine croissance de l'autonomie sectorielle des policiers n'a pas suscité de remous chez les procureurs. Il faut y voir, certes, une classique résignation à l'égard de la puissance policière ${ }^{16}$, mais surtout un effet multiplicateur autour de la CrimOrg lié à l'homologie conjoncturelle parfaite des perceptions policières et judiciaires sur l'extrême gravité, l'urgence, l'ampleur considérable et surtout l'invisibilité du phénomène (pp. 263-265). Cette homologie des définitions de la réalité amène les procureurs, en parfaite conscience, à octroyer à la police une autonomie aussi considérable qu'attentatoire aux droits fondamentaux ${ }^{17}$. Pour le formuler en termes de procédure pénale française, on dirait qu'en réalité la LCO est une enquête de délit flagrant... sans délit, ni borne temporelle, ni matière, ni contrôle. Aussi, si la nature de la LCO est constructiviste, au sens où elle consiste en la poursuite d'un construit policier, ses effets sont loin d'être purs produits de l'esprit: les 1065 enquêtes sous couverture engagées de 1992 à 1995, par exemple, dans le Bade-Wurtemberg ont permis l'interpellation de 1027 auteurs de délits, la saisie de stupéfiants d'une valeur totale de 13 millions d'Euros, de fausse monnaie pour 8 millions, de peintures, d'antiquités et de biens divers pour 12 millions, etc. (p. 111). Efficacité de la lutte contre le crime ? Sans doute. Ces délits relèvent-ils de la CrimOrg ? Rien n'est moins sûr. Et c'est là que le bât blesse. Alors qu'un délit de CrimOrg a été créé (voir plus loin), les peines prononcées restent de criminalité ordinaire ( 3 homicides, 17 hold-up, 5 incendies volontaires, 1 chantage, 1 explosion criminelle) et, ajoutera-t-on, de criminalité très ordinaire (340 vols de véhicules, 350 vols avec effraction, 97 fraudes aux assurances). On peut se rassurer, en un sens : des policiers qui enquêtent, trouvent. Le seul problème est que ce qu'ils trouvent, précisément, est à mille lieues de ce qu'ils partaient chercher. Infraction au principe de légalité, qui veut qu'une intervention répressive soit toujours liée à un délit. Infraction au principe de l'opportunité des poursuites, qui veut que la police enquête, mais ne décide pas de ce sur quoi elle va enquêter. Infraction à la définition de l'enquête judiciaire, ici considérée comme de flagrant délit, alors que l'enquête voit sa nature et son déclenchement fondées sur le fait qu'il n'y a pas eu délit ${ }^{18}$. Tant d'infractions sans sanctions? C'est que même la loi, en Allemagne, a changé. Les lois de 1992 (dite "OrgKGesetz») et de 1994 (dite "de lutte contre la criminalité ») ont introduit, ou plutôt consacré ${ }^{19}$, l'autonomie presque totale de la police judiciaire, dont nous venons de dire les aspects les plus spectaculaires (pp. 180-212). On touche là au point névralgique de la LCO. Prise en ses pratiques singulières, elle ne serait qu'une forme épurée de police judiciaire (marquée en cela par l'autonomie d'intervention, le secret des opérations, la détermination a posteriori des 
moyens légaux ${ }^{20}$, etc.). Désormais, la loi permet de les comprendre comme un tout. La codification a pour but, ici, la consécration en droit de ce qui n'était que pratique judiciaire; ce qui a pour effet (et c'est là mouvement plus singulier) de naturaliser les infractions aux droits fondamentaux que nous avons mentionnées ${ }^{21}$. L'embarras du législateur à codifier peut d'ailleurs être tenu pour un signe tangible des résistances internes à l'entreprise de légitimation par voie légale de l'illicite ${ }^{22}$. Le législateur, dans sa mission codificatrice, doit se conformer strictement aux règles propres de l'exercice, qui exigent que le mot créé désigne une chose pas encore nommée et puisse ainsi faire advenir la chose à l'existence ${ }^{23}$. Or, au début des années 90 , le législateur devait constater, à son grand dépit, que le baptême par la loi avait déjà été joué et rejoué : les art. 244 et 250 du Livre pénal définissent les «crimes commis en bande ", l'art. 129 «l'association de malfaiteurs ». L'entreprise se trouvait alors contrainte à un effort de sur-investissement créatif, plié à la l'impératif visant à figer dans le droit l'invisible forme pure de la CrimOrg. Au final, le texte définit la Crimorg selon le degré de son organisation ${ }^{24}$; que la réalité ne peut jamais démontrer, sauf au terme de ces enquêtes "préventives» sans borne. Aussi, en bout de course de l'exercice codificateur, le policier se voit investi par le texte du mandat de définir a posteriori le phénomène que la loi avait pour ambition de codifier a priori. Produits de débats parlementaires parfois burlesques, captifs d'un mimétisme à l'égard des perceptions policières, existe donc, par la loi, une LCO, qui n'est ni liée à des délits déterminés, ni, donc, à des formes concrètes de manifestation. Pour le dire autrement, et mener le raisonnement du législateur à son terme, la criminalité organisée, n'étant nulle part visible, est partout où on finira par la supposer. Mouvement achevé d'abandon de la loi à une réalité que savoir policier et savoir commun ont faite trop maligne pour se laisser figer dans le droit, la législation allemande sur la CrimOrg peut être tenue pour une forme paradigmatique de remise de soi du législateur à l'institution policière. Voilà qui implique une mutation extraordinaire de la police de LCO. Car si l'objet de la recherche est partout, les moyens de la police ne sont pas infinis. Il faut donc sélectionner : qui sera l'objet de l'enquête préventive? Libérés de la contrainte de l'opportunité des poursuite et de la tutelle des faits, les policiers se lancent en recherches aléatoires auprès de leurs milieux de prédilection. La Crimorg est une affaire d'experts, de ceux qui savent "sentir" les choses (invisibles, de surcroitt, aux yeux du commun). Objets d'enquête les plus fréquents des équipes de LCO : les plus classiques, les plus anciens, les "Rotlicht Milieus ", et les étrangers ${ }^{25}$, ainsi que ceux, de manière plus générale, dont l'apparence affichée tranche avec l'apparence conforme (aux normes de jugement ordinaire des policiers ${ }^{26}$. Et puisque la CrimOrg est sans borne physique ni temporelle, l'enquête sera à la fois des plus intenses et des plus étendues. La CrimOrg est partout. Il faut donc organiser un système de repérage, de collecte et de distribution des données qui soit particulièrement performant. Ont donc été mises sur place, dans les grandes villes, les Länder (Landeskriminalämter - LKA) et au niveau fédéral (Bundeskriminalamt - BKA), des banques de données baptisés APOK, qui ont deux spécificités. La première est que, comme ce qu'elles renferment n'est pas lié à un délit, mais à des personnes ou à des milieux ou à ce qui se fera sentir comme pouvant être de la CrimOrg, ces données vont puiser partout: dans les fichiers sur les délits et personnes liées aux stupéfiants, aux armes, aux délits Est-européens, aux divers fichiers propres aux $\mathrm{LKA}^{27}$, aux fichiers d'enregistrement des adresses ${ }^{28}$, aux fichiers d'enregistrement des étrangers, etc. (pp. 43-44). Comme la CrimOrg n'est pas un délit ponctuel, mais une organisation continue, les fichiers ne sont jamais détruits mais 
conservés éternellement. La seconde de ces spécificités est liée au fait que partout présente, la CrimOrg peut tout aussi bien l'être au sein même de la police. Dès lors, si l'information doit toujours converger vers ces fichiers APOK ( devoir de livraison des données" - Bringepflicht - à l'égard de toutes les administrations détentrices de données), jamais elle ne redescend vers les autres services de police (pp. 42-43). Aussi, si les policiers sont des " knowledge workers ", comme se plaît à le décrire une nouvelle sociologie anglo-saxonne, alors ces policiers LCO sont au sommet de leur monde. Cela se manifeste d'ailleurs par les équipements de réclusion physique à l'égard des collègues, de la séparation architecturale aux cartes magnétiques qui permettent un contrôle des flux à l'intérieur des postes de police (p. 177). Des données sont rassemblées, parfois produites, des enquêtes sont déclenchées (le plus souvent, en fait, sans être motivées par les données enfermées dans les fichiers - dans seulement $22 \%$ des cas, p. 59 - voir plus loin). L'enquête consiste en une surveillance continue de ces gens et milieux traditionnellement cibles de l'action policière : il s'agit de tout savoir, pour tout prévoir. Si, au bout d'un moment, un délit est commis, les policiers décident de passer à l'action selon qu'ils estiment ou non que le délit en appellera d'autres. Donc, le plus souvent, les policiers restent en observation. Comment? Non pas par la mobilisation de moyens techniques ou scientifiques considérables. Les écoutes, par exemple, sont d'un faible rendement (les « chefs de la CrimOrg » ne téléphonent jamais, c'est chose connue - p. 76) ; elles ne constituent qu'un demi millième des enquêtes (p. 76). Les moyens sont classiques, ils relèvent de la police de Fouché : agents infiltrés et indicateurs. Bien surprenant est ce constat en effet: les formes de la CrimOrg se trouveraient à un niveau de scientificité tel que la lutte qu'elle engendre exigerait moyens abondants et technologies de pointe. Certes, les services LCO sont bien mieux équipés que les autres ${ }^{29}$. Mais leurs modus operandi restent canoniques: indics et mouchards. Ce qui impressionnera ici surtout le lecteur, c'est le degré de sophistication du contrôle exercé sur les V-Männer et les verdeckte Ermittler, à mille lieues des formes rudimentaires d'enquête sous couverture en France ${ }^{30}$. Toute une réglementation policière des espions et des mouches est mise en place. Ces derniers, par exemple, s'ils sont dans leur écrasante majorité des déjà condamnés, sont formés au métier de mouche par des policiers, et chacun "travaille " sous la responsabilité d'un policier. Pièces centrales de la LCO, car c'est par leur biais que l'éventuel policier sous couverture (verdeckte Ermittler) sera introduit dans le milieu enquêté, ce sont au final les mouchards qui décident du déclenchement de l'enquête! Pour sa part, le verdeckte Ermittler est plus encadré encore que le non professionnel. Ses qualités doivent être celles du parfait «bureaucrate » (objet d'un contrôle étroit, il doit remplir sans relâche toutes sortes de fiches à l'attention de son chef) et doit "d'un autre côté évoluer tranquille avec cet habitus peinard propre au milieu " (chef d'intervention, BKA ou LKA, p. 103). Tous suivent une formation en LKA ${ }^{31}$, sous l'autorité d'un verdeckter Ermittler Führer, qui veille au contrôle, à l'efficacité, à la durée et à la sûreté de l'agent sous couverture. Les infiltrés doivent rendre compte à leurs supérieurs, être le plus souvent possible, dans leur vie privée, en contact avec des policiers, pour ne pas perdre leur identité et leur loyauté (p. 105). Une professionnalisation accrue du métier d'agent infiltré prend ainsi forme; professionnalisation qu'il faut à la fois entendre comme la forme achevée de la revendication de juridiction des policiers LCO (au sens où elle a pour vœu d'assurer l'étanchéité la plus forte entre V-Männer profanes et verdeckte Ermittler consacrés) et comme la manifestation de la nécessité pour ces policiers de faire de cette nouvelle profession une éthique instituée, celle d'une police propre (au 
sens où les débats sur la surveillance policière en Allemagne sont constamment affectés par le souvenir de la Gestapo et de la Stasi) ${ }^{32}$. Résultat ? On a déjà lu quelques éléments chiffrés, où l'on ne trouve guère ces «gros poissons " qui font l'honneur de la haute police. Les résultats échouent face à la chimère, ou l'hydre, que policiers, procureurs et politiques ont voulu dessiner sous les traits de la CrimOrg. Les chefs tirent les ficelles, de toutes façons, depuis l'étranger. Et il ne faut pas s'attendre à ce que les policiers sous couverture parviennent jamais à monter les niveaux de la hiérarchie de l'organisation criminelle. D'ailleurs, quoi qu'il en soit, la Crimorg est si bien développée qu'elle fait désormais partie intégrante de l'économie... licite (témoignages de policiers, pp. 114-117). Alors que reste-t-il, au final ? Un peu de dépit, beaucoup de bruit ${ }^{33}$, et des policiers soustraits aux procureurs, aux politiques, aux pairs. Des policiers qui surveillent, qui infiltrent, qui notent et qui consignent, ad vitam aeternam, le plus souvent à perte, les mille et une choses des milieux un peu louches, en tous cas mal vus. On pourrait conclure à un changement de régime de l'action policière, comme le fait Pütter à maintes reprises de ce livre excellent, qui voit là la mise en place d'une police proactive, munie de moyens exorbitants. En fait, et l'auteur l'indique aussi (dès la page 15), cette police un peu spéciale ne l'est pas tant. Elle reprend à son compte, pour en consacrer par voie législative la pérennité, ces inclinations structurelles de la police judiciaire : être maître de son agenda, maître de ses moyens, maître de ses objets. Cette petite onction d'aristocratie des policiers LCO, qui les fait les meilleurs de leurs pairs, et pourtant si semblables à eux, n'est ainsi que la manifestation de la pureté du métier qu'ils sont parvenus à consacrer. Se déploie donc sous nos yeux l'institutionnalisation accomplie d'une profession particulière; ici, en un mot, de la police pure.

\section{NOTES}

1. Chronique de l'ouvrage de Norbert Pütter, Der OK-Komplex. Organisierte Kriminalität und ihre Folgen für die Polizei in Deutschland. Münster : Westfälisches Dampfboot, 1998, 450 pages. Toute ma gratitude va à Dominique Linhardt pour ses remarques sur ce texte dont je ne peux que m'attribuer les erreurs et les imperfections. 2. Dominique Monjardet, « Le chercheur et le policier. L'expérience des recherches commanditées par le ministère de l'Intérieur ", in Revue française de science politique, avril 1997, pp. 211-225.

3. Au point que, au sein des bureaucraties policières, la visibilité des moyens de l'action policière, en est devenue la fin même, transformant ainsi dans sa mise en œuvre la substance de la politique publique visant l'action de proximité de la police (phénomène de l'involution des buts que décrit Dominique Monjardet, Ce que fait la police. Sociologie de la force publique. Paris, La Découverte, coll. « Textes à l'appui/ sociologie ", 1996, pp. 214-217). L'abondance de la production scientifique sur ces phénomènes ainsi rendus doublement visibles n'en est donc sans étonnement que plus grande.

4. Carl Klockars, «The Dirty Harry Problem », Annals of the American Academy of Political and Social Science, novembre 1980. 
5. De ce point de vue, un test de la validité de la démarche constructiviste consisterait à savoir des policiers s'ils croient eux-mêmes aux constructions qu'ils élaborent. On serait alors surpris qu'en réalité, ils sont rarement dupes de leurs propres chimères. Pour reprendre les termes de ce chef fédéral de bureau LCO « La criminalité liée à la drogue, on ne la combat jamais mieux qu'en fermant les bureaux de police chargés de la lutte contre la criminalité liée à la drogue. Parce que là, on n'aura plus de criminalité liée à la drogue du tout » (p. 169). Il n'est pas dit non plus que cette expression de cynique réalisme soit dans le même temps celle de la toute-puissance : elle manifeste souvent au contraire l'impuissance à se savoir sous le poids de ce que John Searle appellerait le "fardeau métaphysique de la réalité sociale ", c'est à dire en fait le poids d'une réalité à la fois si intensément faite de mots et si étroitement au contact de la misère sociale (voir par exemple les témoignages désabusés de policiers anti-drogue in Michel Setbon, « Drogue, facteur de délinquance ? D'une image à son usage », in Revue française de science politique, 45, 5, oct. 95, p. 747 et suiv. - et in Pütter, p. 115). 6. Le titre est une manœuvre éditoriale visant à faire de ce livre l'écho du best-seller Der Baader Meinhof Komplex, la riche épopée des quatre membres de la Fraction armée rouge jugés aux fameux procès de Stammheim (1975-77), et dont l'ancien rédacteur en chef de l'hebdomadaire Spiegel, Stefan Aust, est l'auteur (Munich, Knaur, 1989 l'édition originale date de 1985). L'objet de Pütter se laisse bien mieux comprendre, en réalité, par son sous-titre, « La criminalité organisée et ses conséquences sur la police en Allemagne "... La petite ingéniosité éditoriale est d'autant plus trompeuse qu'en réalité c'est moins la lutte contre la RAF qui a marqué les répertoires de LCO que les modes ordinaires de lutte contre le trafic illicite de stupéfiants (p. 88).

7. "Quand on a un crime, c'est un indice certain qu'il ne s'agit pas de CrimOrg » - chef de bureau LCO, commissariat central d'une grande ville, p. 32 .

8. L'exploitation de ces 71 entretiens retient également l'attention. Sur le travail de police secrète, en effet, le lecteur est souvent habitué aux références cursives. Ici, les entretiens sont toujours matière d'appoint à la démonstration, qui chaque fois prend appui sur des sources plus fiables. Mais leur insertion dans le texte est toujours d'autant plus profitable qu'ils sont cités à la forme directe, que le lecteur connait le poste occupé par la personne interrogée, et qu'un extrait est toujours placé en voisinage avec deux ou trois autres, ce qui renforce d'autant mieux la validité de son exploitation scientifique.

9. Voir en ce sens les indications offertes par Ethan Nadelmann sur l'ancienneté de l'importation, en provenance des États-Unis (via le FBI et la FDA), de certaines pratiques d'enquêtes policières, dont celles évoquées ici ( «The americanization of global law enforcement », in William McDonald, Crime and Law Enforcement in the Global Village. Cincinatti : ACJS/Anderson, 1997, pp. 131-136 notamment).

10. On peut en trouver, en français, une bonne description in René Lévy, Du suspect au coupable. Le travail de police judiciaire. Paris, Méridiens/Klincksieck, coll. « Médecine et hygiène ", 1987. En allemand, l'ouvrage, d'une précision considérable, de Stock et Kreuzer sur la lutte contre les stupéfiants, permet lui aussi d'exposer en toute clarté la dynamique de la police judiciaire par rapport à la police de sécurité publique, à l'autorité judiciaire et au législateur (Jürgen Stock et Arthur Kreuzer, Drogen und Polizei. Eine kriminologische Untersuchung polizeilicher Rechtsanwendung. Bonn, Forum Verlag Godesberg, 1996). La lecture de cet ouvrage complète parfaitement celle de Pütter, au regard notamment de l'abondance et de la rigueur des sources utilisées (Stock et Kreuzer, pp. 27-53). 
11. Ce sont les termes de l'article 14 du Code de procédure pénale français.

12. Voir sur ces points Fabien Jobard et Olivier Fillieule, «Action publique sous dépendance. Conditions et effets du changement de paradigme dans la lutte contre la délinquance associée à la drogue en Europe ", Revue française de science politique, 6, 49, décembre 1999, pp. 805-835 et l'ouvrage déjà mentionné de Stock et Kreuzer, p. 73 et suiv.

13. Nous garderons ici le masculin Männer, en dépit de la féminisation croissante des orthographes de noms en allemand : quintessence de la police criminelle, la LCO est par conséquent en majorité écrasante un métier d'hommes.

14. Et ce sont alors les policiers qui se découvrent eux-mêmes constructivistes ! L'enquête démarre « dans le but... je ne dirais pas, de construire, mais disons de trouver l'organisation criminelle » (témoignage d'un policier, chef d'un bureau LCO - p. 50, nous avons souligné).

15. Elle relève alors de ce que l'on appelle en Allemagne le "droit policier ", qui existe en France sous la forme très restreinte, et d'ailleurs tombée en désuétude, de l'enquête préliminaire (cf. André Decocq, Jean Montreuil, Jacques Buisson, Le droit de la police. Paris, Litec, 1991, René Lévy, op. cit., p. 61, Haritini Matsopoulou, Les enquêtes de police. Paris, L.G.D.J., coll. Bibliothèque des sciences criminelles, t. 32, 1996).

16. Voir les remarques fameuses de Philippe Robert sur l'ancillarité de la justice à l'égard de la police criminelle (La question pénale. Paris/Genève, Droz, 1984). Le constat est le même en Allemagne (voir la fameuse étude du Max Planck Institut für Strafrecht de Fribourg : Paul Kerbel, Zur Stellung, Organisation und Tätigkeit der Staatsanwaltschaft. Francfort/Main, 1974.

17. On ne peut malheureusement détailler toute la richesse des descriptions des relations juridiques et pratiques entre policiers et procureurs, que l'on doit là encore lire en parallèle aux observations très riches de Stock et Kreuzer, pp. 232-275.

18. Au point que le droit de la LCO, s'il existait, constituerait matière de choix pour démonstration a contrario de ce que sont les grands principes du droit criminel, dont celui-ci, parmi d'autres : « n'intervenir que dans la limite du nécessaire, mais chaque fois et tant qu'il est nécessaire, voilà l'équilibre difficile à trouver, dans les incriminations, les procédures et le choix des peines. Or la pesée de cette nécessité va se faire à l'aune de la proportionnalité, celle qui doit exister entre la mesure en cause et l'objectif à atteindre "... (Renée Koering-Joulin et Jean-François Seuvic, « Droits fondamentaux et droit criminel », AJDA, juill.-août 1998, p. 116 -souligné par les auteurs. En 1994, le ministère de l'Intérieur fédéral se félicitait qu'un tiers des enquêtes policières pussent déboucher sur des enquêtes judiciaires!

19. Car en réalité la LCO ne fait que renforcer « l'effectivisme policier » sur le modèle hérité de la lutte contre la drogue. Il faut souligner, pour preuve, que la loi de 1992, abrégée OrgKG, porte en réalité pour titre « Loi en vue de la lutte contre le trafic illégal de stupéfiants et d'autres formes apparentes de CrimOrg ", faisant donc des pratiques de lutte contre la drogue un dispositif législatif contre la CrimOrg (sur le volet «stupéfiants » de cette loi, voir Stock et Kreuzer, op. cit., 1995, chap. 5).

20. Sur ce dernier point, voir chez Pütter pp. 241-257 et chez Stock et Kreuzer pp. 257-269.

21. Peut-être est-ce d'ailleurs pour cette raison que les peines prononcées se rapportent en écrasante majorité à des faits de criminalité ordinaire. En Allemagne, la Cour constitutionnelle fédérale peut être saisie a posteriori par voie d'exception... On sait qu'aux Etats-Unis, la décision « H.J. Inc. Versus Nothwestern Bell Telephone Co. » 
(1989) de la Cour suprême considère la définition légale de la CrimOrg comme étant trop vague (voir Robert Luskin, « Behold, the day of judgement. Is the Rico Pattern Requirement void for vagueness? ", St John's Law review, 64, 4, 1990, pp. 779-796, cité in Jean-Paul Brodeur, « Organized Crime. A review of the literature », Annales internationales de criminologie, 35, 1-2, 1997, pp. 89-129.

22. L'embarras n'aura pas été moins grand aux Etats-Unis et au Canada, si l'on en croit le papier de Brodeur cité en note précédente. En Italie, ce sont 114 lois sur la CrimOrg qui ont été promulguées de 1982 (année de l'assassinat du chef du PC sicilien Pio La Torre et du préfet Della Chiesa) à 1992, dont l'application pénale, du fait de leur imprécision, a, jusqu'en février 1992, trouvé pour obstacle majeur la Cour de cassation italienne elle-même (cf. Umberto Santino, « Fighting the Mafia and organized crime. Italy and Europe », in William McDonald, 1997, op. cit., pp. 152-156 notamment). 23. Sur ce point, voir notamment Anton Schütz, « L'immaculée conception de l'interprète et l'émergence du système juridique. A propos de 'fiction' et de 'construction' en droit », Droits, 21,1995, Fernando Acosta, Alvaro Pires, "Constructivisme versus réalisme. Quelques réflexions sur les notions de crime, déviance et situations problématiques ", in Politique, police et justice au bord du futur. Mélanges pour et avec Lode van Outrive Paris : L'Harmattan, col. Logiques sociales / Déviance et société., 1998, pp. 40-41 notamment et Michael Corsten, « Between constructivism and realism. Searle's theory of the construction of social reality ", Philosophy of the social sciences, 28, 1, mars 1998, pp. 104-110. Dans un article récent, Francoise Tulkens et Michel van de Kerchove écrivent : «si les tendances les plus récentes devaient se poursuivre, il semble probable qu'on aboutirait aux résultats exactement inverses de la codification, à savoir une diffraction de plus en plus polymorphe du pénal aussi bien au niveau infra que supraétatique » ("D’où viennent les flous du pénal ? », in Politique, police et justice... op. cit., 1998, p. 133). Ils voient dans ce mouvement une contradiction essentielle posée à l'entreprise même de codification, qui serait, à l'image du projet de réforme du Code pénal, en péril. L'exemple de la LCO incite au contraire à penser que la syntaxe de la codification, tendant à sa compromission avec l'ordre des faits, sauve l'œuvre codificatrice comme moment achevé des processus d'institutionnalisation (constat qui se pose en parfaite contradiction, notamment, avec la perception quelque peu rigide de la codification et de la « force propre du droit » chez Pierre Bourdieu, in « La force du droit. Eléments pour une sociologie du champ juridique », Actes de la recherche en sciences sociales, 64, 1986.

24. En bout de course, l'organisation visée dans la loi sera l'organisation organisée d'une « autre manière » que la bande ou l'association de malfaiteurs... (p. 211), ou bien "à la manière d'une bande ", ou bien tout simplement d'une " association de malfaiteurs » (p. 188). En somme, la criminalité organisée sera organisée (p. 188). 25. Le constat d'un bris de fenêtre dans les toilettes d'un restaurant n'est pas matière à déclenchement d'une enquête en LCO. Sauf s'il s'agit d'une pizzeria... (témoignage d'un chef de bureau LCO, BKA ou LKA - p. 38). Ce renforcement de la surveillance légale des étrangers aura également pour conséquence la consolidation de la criminalité enregistrée des étrangers, et pourra au besoin marquer un nouveau palier dans les constructions rhétoriques soutenant les dispositifs législatifs et réglementaires de lutte contre la délinquance perpétrée par les membres des minorités visibles (voir l'analyse du dernier rapport de la BKA sur les chiffres de la délinquance in Oliver Brüchert, « Die 
Ausländerkriminalität sinkt nicht ! Der Zusammenhang von Kriminalstatistik und Rassismus », Bürgerechte und Polizei, 65, 1, 2000, pp. 21-28).

26. Ce délit d'infraction au sens de l'ordre social ordinaire n'est là encore pas une propriété neuve de l'action policière (cf. Fabien Jobard, « Le banni et l'ennemi. Le travail quotidien de la police à l'égard des marginaux et la notion d'ennemi intérieur ", in VIè Congrès de l'Association française de science politique, Rennes, 26-28 septembre 1999). Ce qui est nouveau, ici, est que la procédure pénale tend à valider ce modus operandi par la notion de " train de vie injustifié ». Et les moyens d'enquête associés à ces poursuites rendent ce délit d'apparente déviance plus dangereux encore.

27. Chaque LKA dispose de ses propres critères de définition de la CrimOrg...

28. Quiconque emménage doit signaler sa nouvelle résidence à l'administration chargée des emménagements (Einwohneramt). Cette institution, objet d'éclats répétés de l'extrême-gauche allemande dans les années soixante-dix et quatre-vingts, avait joué un rôle moteur dans les enquêtes liées à la Fraction armée rouge (cf. Aust, op. cit., 1989).

29. Preuves que les policiers LCO ne sont pas comme les autres : ils ne se plaignent jamais de carences en matériels et équipements (p. 172) et font ainsi injure à un trait les plus constants de la sociologie des organisations policières !

30. Voir la lecture de l'impressionnant récit de Michel Naudy et Dominique Loiseau (L'affaire Loiseau. Le dossier noir de la police des polices. Paris : Plon, 1994) par René Lévy et Dominique Monjardet in « Undercover policing in France », in Gary Marx et Cyrille Fijnaut (dir.), Undercover. Police Surveillance in International Perspective. La Haye : Kluwer Law International, 1995, pp. 29-54.

31. Seuls la Sarre, Berlin et Brême n'ont pas de verdeckte Ermittler : ils les empruntent aux centrales d'enquêtes sous couverture des autres Länder (p. 109).

32. On ne peut ici malheureusement ouvrir le débat, qui se fait urgent, sur ce que signifie précisément la professionnalisation policière, notamment si on veut l'entendre selon la terminologie étroite que donne la sociologie anglo-saxonne à ce terme (dans le débat, pourtant absent de la sociologie de la police, qui oppose « profession » à " occupation »). On se contentera ici de souligner que la codification du sale boulot (l'inflitration) concourt certes à la monopolisation de cette police LCO sur un domaine épistémologique donné, la CrimOrg (c'est ce que résume la notion de « revendication de juridiction » chez Abbott, A., The system of professions. An essay on the division of expert labor. Chicago : The University of Chicago Press, 1988), mais répond dans le même temps à des nécessités auto-produites d'équipement institutionnel de valeurs morales bien précises (on rejoint là la professionnalisation entendue contre Abbott par Halliday, T., Beyond monopoly. Chicago : University of Chicago Press, 1987).

33. La Crimorg, notamment de l'Est, et si possible russe, est un thème chéri de la presse à plus ou moins grand tirage, des boulevardiers Bild, BZ et Berliner Kurier (ces deux derniers, du fait de leur lectorat régional, spécialisés sur les mafias russes du Kurfürstendamm à Berlin) aux journaux plus établis comme Der Spiegel. 
INDEX

Mots-clés : criminalité, lutte contre la criminalité organisée, mafias et crime organisé, police

Thèmes : Bundeskriminalamt (BKA) 